\title{
Central Nervous System Involvement in Lyme Disease - Making the Diagnosis and Choosing the Correct Treatment
}

\author{
Ruxandra Calin ${ }^{1,2}$, Adriana Hristea ${ }^{1,2}$ and Radu Tanasescu ${ }^{2,3}$ \\ 1 Professor Dr. Matei Bals National Institute of Infectious Diseases, Bucharest, \\ ${ }^{2}$ Carol Davila University of Medicine and Pharmacy Bucharest, \\ ${ }^{3}$ Department of Neurology, Colentina Hospital, Bucharest, \\ Romania
}

\section{Introduction}

Lyme disease is a tick-borne systemic illness caused by three pathogenic species of the spirochete Borrelia burgdorferi sensu lato (B. burgdorferi sensu stricto, B. afzelii and B. garinii). They are flagellated bacteria close to Treponema pallidum, the ethiologic agent of syphilis. All three pathogenic species occur in Europe, Ixodes ricinus being the tick responsible for their transmission in this area. Two of the Borrelia species (B. afzelii and B. garinii) have been identified in Asia. B. burgdorferi is the sole cause of the disease in the United States. Although in United States the proportion of neuroborreliosis among Lyme disease manifestations is of about $8 \%$, in Europe the incidence of this form of disease is more elevated $16-46 \%(1)$.

Lyme disease has a broad spectrum of clinical manifestations, and variable degrees of severity due, in part, to differences in the infecting species. In France, for example, neurologic manifestations of Lyme disease are frequent due to the endemic presence of $B$. garinii (2). Historically, the first case of neuroborreliosis in medical literature was described in 1922 in Lyon: meningoradiculitis, preceded by a tick bite and migratory erithema, in a patient who presented a false positive syphilis serology. Meningoraticulitis remains even today the most frequent form of neurolyme, even though the known spectrum of disease can be very broad, varying from mild impairment to severe, cognitive or even psychiatric manifestations. Phenomenology-based assertions that "Lyme disease can do anything" in the nervous system or that Lyme disease is the new "grand imitator", make difficult the task of differentiating between true nervous system disorders and the effects of extra-neurologic disease on behavior and cognitive function.

In this section the neurologic manifestations of Lyme disease, with special emphasis on Lyme meningitis, encephalomyelitis will be reviewed. The definition of a controversial entity - Lyme encephalopathy - will be discussed. Much of the confusion and controversy surrounding Lyme disease relates to misunderstandings about what does and does not constitute evidence of nervous system infection. Emphasis on several nonspecific symptoms in early studies $(3,4)$ led to sometimes erroneous conclusions regarding symptoms specific 
for Lyme disease. This misunderstanding was one of the elements that contributed to debatable attitudes regarding which individuals required treatment for Lyme disease, what constituted effective treatment, and how to define cure (5).

In order to have an accurate understanding of nervous system Lyme disease the true scope of nervous system disorders attributable to Lyme disease and their corresponding pathophysiologic mechanisms, need to be better defined.

\section{Epidemiological background}

Epidemiological context is essential to establishing the diagnosis of Lyme disease. Even so, it is not always easily highlighted.

Following the infestation of a tick during a meal on an infected mammal, Borrelia will develop inside the acarian and will accidentally be transmitted to a human host. Transmission will mostly occur in a humid environment (forest, field, even garden). The risk of transmission is proportional to the duration of tick attachment: at least 12 hours are necessary in order for the Borrelia to reach the salivary glands of the tick. The bite of Ixodes ricinus is painless and the larva tick measures up to $1 \mathrm{~mm}$. Consequently tick bites are discovered in only $26-38 \%$ patients $(6,7)$.

Few studies have looked into the different clinical patterns seen with different Borrelia species. Strle et al has shown in a study of 33 european patients in which Borrelia could be isolated in culture, that B. garinii was mostly associated with meningitis symptoms $(61 \%$ for B. garinii versus $10 \%$ for $\mathrm{B}$. afzelii, $\mathrm{p}=0,009)$ and painful radicular symptoms $(65 \%$ versus $0 \%, \mathrm{p}<0.001$ ), while neurocognitiv impairment seemed more frequent in patients infected with B. afzelii, even though statistical significance was not proven (8).

There are two age incidence peaks: in childhood and between 50-60 years of age. Males are 1,5 times more often infected than females. The first manifestations of the diseases develop most often in spring-autumn, especially in June - November $(6,7)$.

The early, localized phase, occurring a few days to one month after the tick bite consists in migratory erythema. The diameter of the lesion can range from a few millimeters to tens of centimeters. It is mostly annular, but can be polymorphe and can be easily mistaken for the local inflammatory reaction at the site of the bite or can remain unperceived (9). Consequently migratory erythema is noted in less than half of the patients (18-46\%) (9). Associated symptoms and signs during this early, localized, stage may include: fatigue, malaise, lethargy, mild headache, mild neck stiffness, myalgias, arthralgias and/or regional lymphadenopathy.

\section{Clinical spectrum of neuroborreliosis}

Neuroborreliosis is found either in the context of an early disseminated disease or during late, chronic Lyme disease. The clinical features of nervous system Lyme disease, although proteiform?, have certain characteristics. Clinical features of Lyme disease may be a result of direct bacterial infection (particularly in the early stages of disease) or a consequence of the immune response leading to symptoms in different organs. 
Just as in neurosyphilis, nervous system involvement begins during early disseminated Lyme disease, when spread of the spirochetes can result in meningeal seeding (10). As in syphilis, untreated Borrelia infection probably subsides in some patients but becomes chronic in others. Also as in syphilis, appropriate antimicrobial treatment results in microbiologic cure in the overwhelming majority of patients, regardless of the duration of the infection.

Neurologic features of early disseminated Lyme, developing at weeks to months after tick bite disease may include:

- $\quad$ Lymphocytic meningitis

- Unilateral or bilateral cranial nerve palsies (especially of the facial nerve)

- Radiculopathy

- Peripheral neuropathy

- Mononeuropathy multiplex

- Cerebellar ataxia (rarely)

- Encephalomyelitis (rarely)

They can occur in the absence of any prior features of Lyme disease. The classic triad of acute neurologic abnormalities is meningitis, cranial neuropathy, and motor or sensory radiculoneuropathy, although each of these findings may occur alone (5).

Although the facial nerve is the most commonly affected cranial nerve, other nerves such as the abducens nerve may be involved. Lyme disease is one of the few causes of bilateral cranial nerve palsies. Other causes include tuberculosis, sarcoidosis, and trauma.

The neurologic manifestations of late Lyme disease are different from those in early disseminated disease. They occur month to years after tick bite and in the absence of any prior features of Lyme disease and only about 25 percent of patients with erythema migrans recall the tick bite that transmitted Lyme disease $(6,7)$. In the United States, a mild neurologic syndrome, called Lyme encephalopathy, has been reported, manifested primarily by subtle cognitive disturbances $(11,12)$. In the United States and Europe, a chronic axonal polyneuropathy may develop, manifested primarily as spinal radicular pain or distal paresthesias $(7,11,12)$. In Europe, B. garinii may rarely cause chronic encephalomyelitis, characterized by spastic paraparesis, cranial neuropathy, or cognitive impairment with marked intrathecal antibody production to the spirochete (7).

It is unknown whether the time course of these syndromes is related to differences in spirochetal strain, inoculum size, or host response. However, the syndromes associated with abrupt onset typically occur early in infection (within the first few months), whereas those that are more indolent and protracted often present later in the course (eg, many months after initial infection) (5). Consideration of the clinical findings of nervous system Lyme disease is divided into disorders of the peripheral versus central nervous systems. In both, the predominant mechanism appears to be multifocal inflammatory involvement of the affected structure, be it patchy peripheral nerve involvement or, rarely, patchy inflammation of the central nervous system.

Regardless of the clinical syndrome, a frequently found accompanying feature of disseminated disease is radicular pain, reported in $38 \%$ to up to $86 \%$ of patients with 
neuroborreliosis $(6,7,9)$. Headache is also found in $18-43 \%$, neck stiffness is almost exlusively found in children, while arthralgias are described in only $1-18 \%$ of patients and myalgias are rarely found, in up to $13 \%$ patients with neuroborreliosis $(6,7)$. Fever is noted in $10 \%$ of patients. Other very rare manifestations are: myocarditis with atriventricular block $(1 \%)$, atrophic acrodermatitis (dermatologic features of chronic diseases, found in less than $1 \%$ of patients with neuroborreliosis, in large cohorts) and chorioretinitis $(6,7,9)$.

\section{Acute meningoradiculitis and meningitis}

The two largest European studies published to date, on a total of 187 patients (Hansen et al, Danmark) and 330 patients (Oschmann et al, Germany), reported meningoradiculitis as the predominant form of neuroborreliosis (with an incidence of $67 \%$ and $85 \%$ respectively) $(6,7)$. The delay between the tick bite and the neurologic syndrome is, in average, 3 weeks, but can extend to more than three months (6). The typical, but non compulsory, sequence is: migratory erythema at 3 days after the bite, sensitive radiculitis 3 weeks after, than paresis in the two following weeks, with or without the apparition of new radicular impairment (6). In the European literature, involvement is said to parallel the site of the tick bite (14); this has not been addressed systematically in the United States. Pain is often a prominent element, the hallmark of the syndrome. It can be described as severe, "burn", "tear" or painful paresthesias, more accentuated at night and preventing sleep (9). Pain is not always confined to strict radicular topography, is accompanied by hypo/disesthesia, local or diffuse and is not easily alleviated by usual painkillers, including opiods (9). Only antibiotic treatment by ceftriaxone can lead to a resolution of the symptoms. This disorder can mimic a mechanical radiculopathy (eg, sciatica) with radicular pain in one or several dermatomes, accompanied by corresponding sensory, motor and reflex changes, but the presence of such deficits is not constant, even in the presence of subjective complaints. Lyme disease should be considered in patients in endemic areas presenting in spring through autumn with severe limb or truncal radicular pain without an apparent mechanical precipitant (5). In European neuroborreliosis, the term Garin-Bujadoux-Bannwarth syndrome (or Bannwarth syndrome) has been applied to the constellation of painful radiculoneuritis, often accompanied by meningitis with minimal headache and sometimes with cranial neuropathy (15).

In more than $50 \%$ of cases meningoradiculitis are accompanied by cranial nerves involvement (7). The spectrum of Lyme cranial neuropathies will not be detailes here, but facial nerve involvement represents about 80-90 percent of all Lyme disease-associated cranial neuropathies (6). Since facial nerve palsy is uncommon in children, Lyme disease should be strongly considered as the cause of facial nerve palsy affecting a child who has been in an endemic area. In adults in endemic areas, during spring through fall, a significant percentage of facial nerve palsies are attributable to Lyme disease. In one study in an endemic region, one quarter of cases of facial nerve palsy in the summer were likely due to Lyme disease, based upon serologies (16). Involvement can be bilateral, with onset being either simultaneous or in rapid succession. Because bilateral facial nerve palsies are generally uncommon, such cases should bring Lyme disease to mind in patients with potential recent exposure. The differential diagnosis of bilateral facial nerve palsies is limited and includes sarcoidosis, Guillain-Barré syndrome, HIV infection and other basilar meningitides (5). 
Cerebrospinal fluid analysis is essential to the diagnosis of meningoradiculitis, but the findings are non specific. Most often there is increased cellularity, with lymphocytic predominance, similar to viral meningitis. Hyperproteinorahia (frequently around $1 \mathrm{~g} / \mathrm{l}$, but can be normal or as elevated as $5 \mathrm{~g} / \mathrm{l}$ ) and IgG oligoclonal bands can be found in $70 \%$ of cases (7). When accompanied by peripheric involvement, axonal damage can by proven by EMG. MRI can show enhancement of spinal or cranial nerve roots. Usually though the clinical picture and the lumbar puncture are sufficient for guiding the diagnosis (9).

The clinical spectrum can include several atypical forms as: ataxic sensitive neuropathy with meningitis, multiple neuropathy with meningitis or motor polyradiculoneuritis with meningitis (9).

Isolated meningitis represents only $4-5 \%$ of neuroborreliosis and is more frequently found in children $(6,7)$. In this setting, headache is rarely severe and the meningitic syndrome, if present, is never as serious as in purulent meningitis. Fever is reported in $4-10 \%$ of patients with isolated meningitis and migratory erythema in only $17 \%$ of cases (7).

Due to their quiet clinical appearance, these acute meningitis forms can remain undiagnosed and progress to chronic infection. The term "chronic" is used when the duration of symptoms extends beyond 6 months, in which case other organic symptoms, such as weight loss, can be present $(7,9)$.

CSF study in isolated meningitis has the same features as in meningoradiculitis. Pleiocytosis is around $200 / \mathrm{mm} 3$ and the presence of oligoclonal bands is less frequent (around only 22\% of cases) (7). Hypoglycorahia can be an important finding, raising the need for a differential diagnosis with tuberculosis. In this context of nonspecific findings, the essential diagnostic tool is the intrathecal anti-Borrelia antibody index (17), which will be described later in this section.

Interestingly, certain tick species are able to transmit both tick-borne encephalitis (TBE) and Lyme borreliosis. Therefore, it is possible that a patient can simultaneously be infected with the TBE-virus and Borrelia burgdorferi-spirochete as a result of a single tick bite. Although this is a rare event, its possibility has to be taken into account, since only neuroborreliosis beneficiates from a specific treatment

Benign intracranial hypertension - A pseudotumor cerebri-like picture (benign intracranial hypertension) occurs with increased frequency in children with Lyme disease $(18,19)$. Virtually all of the reported cases have had inflammatory cerebrospinal fluid, suggesting that the increased intracranial pressure is a result of meningitis, in contradistinction to the usual picture of pseudotumor with a normal cerebrospinal fluid profile. Regardless of the mechanism, the symptoms and potential consequences are identical, including compression of cranial nerves with a particular threat to the optic nerves and vision (5).

\section{Acute myelitis}

In the two large European cohort studies mentioned above acute myelitis are reported in 4$5 \%$ of cases $(6,7)$. They are associated in $35 \%$ of cases with a cranial radiculopathy and in $59 \%$ of cases with spinal neuropathy. Fever is present in $6 \%$ of cases. The medullar syndrome includes: paraparesis ( $75 \%$ of cases), proprioceptive ataxia (35\% of cases), urinary 
dysfunction (20\% of cases) (7). Although reported in a majority of cases, CSF pleyocytosis is not a constant finding. There are only 12 published cases describing a medullar MRI (20). By the time this localization is proven, it usually involves more than three metamers. The localization is most often cervical, high thoracic or cervico-thoracic, but the exact pattern can be variable (most cited: transverse myelitis and predominantly posterior myelitis) (20). Sometimes, when myelitis is not proven on MRI, leptomeningeal enhancement, coupled with clinical data, will orient towards the diagnosis.

\section{Acute encephalitis}

Acute encephalitis and meningoencephalitis represents only $0,5-8 \%$ of neurolyme in Europe $(6,7)$. Headache is present in two thirds of cases. In $40-50 \%$ of patients radicular pain, cranial neuropathies (mostly VI and VII), as well as fever, are part of the clinical picture (7). On the other hand, in $57 \%$ of cases encephalitis presents as acute neuropsychiatric disease: cognitive of memory impairment, disorientation, depression or sleep disorder. In $21 \%$ of cases cerebral ataxia has been described. An asymmetric extrapyramidal syndrome is present in $21 \%$ of patients and hemiparesis in $7 \%$ of cases (7). The consciousness level is rarely and only mildly impaired.

CSF analysis shows lymphocytic pleyocytosis than can be modest, of only a few cells. Proteinorahia is less often elevated and can be normal. The presence of IgG oligoclonal bands in CSF is proven in up to $55 \%$ of cases (7).

Noteworthy, the electroencephalogram (EEG) is abnormal in all patients: a moderate slowing with generalized slow waves with or without spikes is most often found. In $14 \%$ of patients slow theta-delta waves can be found (7). Seizures are present in up to $4 \%$ of patients. The cerebral CT is normal in most cases, as is the MRI. Hypersignal T2 temporal or brainstem lesions have been described (7).

\section{Acute optic neuropathy}

This is an extremely rare manifestation of Lyme disease, which can be circumscribed to the spectrum of neuroborreliosis. In a study of 440 patients with optic neuropathy, Sibony et al found the presence of Lyme disease in only 2 patients (21). There are only 2 other cases of isolated Lyme optic neuropathy described in literature. Nine other cases of associations between optic neuropathy and other neurologic features of Lyme disease have been reported. In the majority of these cases a positive intratechal anti-Borrelia antibody index was found, accompanied in less than half of the cases by CSF pleyocytosis. The antibiotic treatment led to the amelioration of visual symptoms, even when a delay of a few months existed between the clinical onset and the initiation of treatment $(22,23)$. Lyme disease as a cause of optic neuropathy may be underdiagnosed.

\section{Cerebrovascular disease}

Presenting as both acute and chronic manifestations, cerebrovascular disease is rare, around $1 \%$ of neurolyme cases (7). Ischemic disease, transitory or not, seems to be more frequent, preceded by symptoms of progressive encephalopathy with headache, cognitive impairment and behavioral changes or cranial neuropathy (7). In a recently described case 
of ischemic neuroborreliosis the patient complained for two years of acustic impairment, for which no etiology was found (24). Ischemic lesions seem to be more frequently localized in the basal ganglia, the vertebrobassilar system or the subcortical regions. Diffuse or local stenosis can be proven on angio MRI $(7,24)$. In this context CSF study will show a lymphocytic pleyocytosis with hyperproteinorahia and oligoclonal bands.

Hemorrhagic vascular lesions have been described in only a few case-reports: 4 cases of subarachnoid hemorrhage, 3 cerebral and 1 medullary, 2 cases of intracerebral hematomas and one atypical case associating myelitis and meningeal hemorrhage (20). One pathology study showed an important lymphocytic perivascular infiltrate, rich in $\mathrm{T}$ lymphocytes, which could provide a possible explanation for a presumptive vascular damage leading to ischemic or hemorrhagic consequences (25).

\section{Chronic encephalomyelitis}

The chronic forms of neuroborreliosis are rare and are preceded by acute unrecognized and untreated Lyme diseases. They represent $4-6 \%$ of neurolyme cases in Europe $(6,7)$. The clinical spectrum can range from a progressive myelopathy to an encephalitic form manifested by seizures, focal deficit or cerebellar syndrome (9). Without treatment, there is no remission in the majority of cases. Medullar syndromes are most frequent $(74-100 \%)$, associated with proprioceptive ataxia (63-100\%), urinary dysfunction (37-62\%) and/or para/tetraparesis (63-100\%) (6). The chronic encephalitic form is rarer: cerebellar syndrome (26\%), cognitive impairment/psychiatric symptoms (16\%), hemiparesis (11\%) (6).

Like the other nervous system forms of Lyme, this chronic encephalomyelitis is frequently accompanied by cranial neuropathy (32-75\%): II, VI, VII, VIII usually implicated. Unlike acute encephalitis, chronic encephalomyelitis can involve the optic nerve $(6,9)$. Also, neurosensorial hearing loss is described in 6 patients with chronic Lyme disease in the study of Hansen et al.

CSF analysis shows constantly a hyperproteinorahia (in average of 3,5g/l, but potentially as elevated as $10 \mathrm{~g} / \mathrm{l}$ ), associated with lymphocytic pleiocytosis (7). In $70 \%$ of cases cerebral MRI is abnormal, showing subcortical or brainstem inflammatory lesions. Meningeal enhancement can also be found, sometimes as the sole finding. Rarely, lesions can be mistaken for multiple sclerosis (1).

\section{Lyme encephalopathy - A controversial entity}

The notion of Lyme encephalopathy is mostly exclusively found in American medical literature. The term was labeled in early studies that found that many patients with Lyme disease described fatigue, cognitive slowing, and memory difficulty (3). However, these symptoms are nonspecific and are frequent concomitants of many inflammatory disorders. They are not, in isolation, diagnostic of either Lyme disease or brain infection. In fact, the term "encephalopathy" was used to describe this state, since this term is most often used to indicate alterations in brain function due to non-neurologic causes. Subsequent work demonstrated that only in rare instances were these symptoms associated with brain infection (ie, encephalitis). In such cases, the diagnosis was readily confirmed by 
cerebrospinal fluid abnormalities and brain MRI findings $(3,12,26)$. According to experts, in the absence of such objective evidence of brain infection, these symptoms should not be considered evidence of nervous system infection with B. burgdorferi.

Clinically, most often cited are: memory impairment (anterograde or retrograde), attention deficit, sleeping disorders (30\%) and profound asthenia (70\%) (27). Depression and/or irritability can be associated. Half of patients complain of headache. The neurologic exam in usually normal or signs of chronic axonal polyneuropathy can sometimes be found. Recently five case of dementia, with important impairment of mnesic and executive functions, have been reported in which a positive intratechal anti-Borrelia antibody index was found, in the absence of any other etiologic explanation (9).

In those instances where nervous system infection can be demonstrated, standard antimicrobial therapy is effective. Multiple studies have shown that prolonged antimicrobial treatment offers such patients no lasting benefit but carries significant risk $(28,29,30)$.

Post Lyme disease syndrome versus chronic Lyme disease - A particular challenge has been the sizable population of individuals labeled as having "chronic Lyme disease" on the basis of nonspecific symptoms including persistent musculoskeletal pain, cognitive problems, and fatigue (31). In a minority of patients, these symptoms have followed microbiologically successful treatment of Lyme disease, a condition termed "post Lyme disease syndrome". However, all objective evidence indicates that these individuals, while suffering from debilitating symptoms, do not have chronic infection with B. burgdorferi and that antibiotic treatment is not beneficial (30). In its typical usage, "chronic Lyme disease" includes the post-Lyme disease syndrome, as well as illnesses and symptom complexes for which there is no convincing scientific evidence of any relationship to B. burgdorferi infection (5) and that are common enough in general population. The relationship between neuroborreliosis and cognitive impairment still remains to be proven and analyzed in prospective studies.

\section{Approach to diagnostic confirmation}

The diagnosis of central nervous system Lyme disease rests on three elements:

- Possible exposure to Ixodes ticks, the only species capable of disease transmission

- Objective evidence of central nervous system Lyme disease

- Laboratory testing (positive Lyme serologies with positive cerebrospinal fluid [CSF] Lyme antibodies) which supports the diagnosis

A positive blood Lyme serology does not always imply that the patient has an active Lyme disease. This serology shows that the patient has been in contact, at some time point, with the B. burgdorferi sensu lato (9). It does not mean that the microorganism has developed any pathogenic effect. In the general, healthy population the prevalence of positive Borrelia serology ranges between 3,3\%-6\%, depending of the study. In this context positive blood Lyme serology allows only an orientation for the diagnosis (32).

Moreover the different laboratory techniques, with different reference values and variable intra-patient, inter-kit and inter-laboratory reproducibility, can sometimes be misleading. 
In order to prevent these serologic diagnostic pitfalls, the US Centers for Disease Control and Prevention, recommends the "two-tier strategy", which is uses a sensitive enzymelinked immunosorbent assay (ELISA) followed by a Western blot (5). An immunofluorescent assay (IFA) may be substituted for the ELISA. If the ELISA is positive or equivocal, then the same serum sample should be tested by Western blot (IgM and IgG immunoblots if early disease is suspected; IgG Western blot alone if late disease is suspected). If the ELISA is negative, the sample needs no further testing.

With the exception of the first four to six weeks of infection, when the specific immune response may not yet have developed sufficiently to provide a measurable antibody response, serologic testing using the two-tier approach for antibodies to B. burgdorferi is highly sensitive and specific for the detection of antibodies.

In patients with suspected central nervous system Lyme disease (ie, Lyme meningitis or encephalomyelitis), testing the CSF for intrathecal production of antibodies to $B$. burgdorferi is useful for establishing the diagnosis. This test is not applicable if involvement is limited to the peripheral nervous system. Even in central nervous system disease, the sensitivity remains unclear. A negative test for Lyme antibodies in the CSF does not exclude central nervous system Lyme disease. Rare cases with negative blood tests and positive CSF have been described, this possibly being due to very recent exposure (33). However such cases should be interpreted with caution.

As in other infections, in nervous system Lyme disease, specific B cells may migrate to the central nervous system and proliferate locally, resulting in intrathecal production of antibodies to B. burgdorferi. However, even in the absence of nervous system Lyme disease, a substantial proportion of $\mathrm{B}$. burgdorferi-specific antibodies diffuse from the serum to the CSF. Therefore, the presence of B. burgdorferi-specific antibodies in CSF does not itself establish the presence of central nervous system Lyme disease or prove that there is local production of antibodies (5).

CSF B. burgdorferi antibody measurement is highly specific for nervous system Lyme disease, with cross-reactions occurring primarily in neurosyphilis (5). However, neurosyphilis usually results in production of reaginic antibodies in the CSF, such as those measured in the venereal disease research laboratory (VDRL) test, which is not the case for Lyme disease. Thus, differentiating between these two spirochetal infections is generally easy.

Determination of test sensitivity is far more challenging, primarily because the studies that have addressed this issue have used quite different patient populations $(17,34,35)$. In Europe, sensitivity for detecting anti-Borrelia antibodies in CSF is reportedly over 90 percent (33). Similarly, in some studies of Lyme meningitis from the United States, sensitivity was approximately 90 percent (36). However, in a more heterogeneous population of patients from the US, sensitivity was lower (34). In a patient with a clinical picture suggesting Lyme disease, a positive study from a reputable laboratory should be viewed as significant evidence in favor of the diagnosis (36). However, negative CSF studies should not eliminate neurologic Lyme disease from consideration if clinical circumstances support the diagnosis.

The existing consensus is that the most appropriate test that allows with a specificity of more than $95 \%$ to attribute a neurologic syndrome to Lyme disease is the intrathecal anti- 
Borrelia antibody index. This index has a sensitivity of $75 \%$, which is superior to culture and PCR. It implies that a blood and CSF sample be collected at the same time (17). A reference laboratory must indicate if the index is positive (in favor of neuroborreliosis), negative or intermediary. An European consensus exist for the utilization of this index in terms of specificity and sensitivity, in order to prevent inter-laboratory variables $(17,33,37)$.

In clinical practice, the first exam to be performed is Lyme blood serology. If positive or if negative in the presence of a highly evocative neurologic syndrome, lumbar puncture should be performed in order to determinate Lyme CSF serology and the intrathecal antiBorrelia antibody index (17). In children, in the presence of facial paralysis with positive blood Lyme serology, lumbar puncture is not necessary (9). The usefulness of the intrathecal anti-Borrelia index in uniquely peripheral forms of Lyme disease, has not yet been established.

Although CSF or serum B. burgdorferi antibody concentrations typically decline after treatment, these antibodies may persist for 10 or more years following successful treatment $(38,39,40)$. Thus, determination of active neuroborreliosis requires combining these results with less specific measures of active central nervous system inflammation (ie, a CSF pleocytosis and/or elevated protein).

As in other infections in which there is a prominent antibody response, when nervous system Lyme disease has been present long enough for the B cell response to mature, there are often increases not only in specific anti-B. burgdorferi antibodies but also in nonspecific immunoglobulin concentrations. Consequently IgG synthesis rate or IgG index can be elevated, and, particularly in patients in Europe with central nervous system Lyme disease, oligoclonal bands can be seen in the CSF (40). The frequency with which these findings are found has varied widely among series. The important point is that oligoclonal bands and increased IgG synthesis occur in infections including central nervous system Lyme disease, and do not support a differential diagnosis with multiple sclerosis.

Other diagnostic tests have been proposed, but are seldom used.

Polymerase chain reaction - The polymerase chain reaction (PCR) for B. burgdorferi can be performed on CSF, although it has low sensitivity (41) probably reflecting the very low number of organisms typically present in spinal fluid. Between this low sensitivity, and a tendency for some laboratories to have difficulty avoiding false positives, the positive and negative predictive values both tend to be low, rendering this test of marginal utility. For that reason, PCR is not recommended for routine testing of CSF in Lyme disease

CXCL13 - Although elevated concentrations of CXCL13, the chemokine B lymphocyte chemoattractant, are not unique to active nervous system Lyme disease, when combined with other diagnostic information, they might serve as a marker of disease activity or response to treatment (42). Like patients with neurosyphilis (43), patients with acute untreated nervous system Lyme disease and a CSF pleocytosis have elevated CSF concentrations of CXCL13 (44). Among 17 such patients, concentrations of CXCL13 were proportionately greater in CSF than in serum and were highly elevated in all CSF samples compared with the CSF of 178 controls without nervous system Lyme disease (mean 15,149 $\mathrm{pg} / \mathrm{mL}$ versus $247 \mathrm{pg} / \mathrm{mL}$ ) (44). Five patients who had received at least two weeks of treatment for nervous system Lyme disease did not have substantially elevated CXCL13 
concentrations (mean $202 \mathrm{pg} / \mathrm{mL}$ ), whereas five patients who had been treated for less than two weeks had slightly elevated CXCL13 concentrations (mean $1412 \mathrm{pg} / \mathrm{mL}$ ). Among the 178 controls, seven had an elevated CXCL13 concentration, five with CNS lymphoma and two with bacterial meningitis. Relapsing-remitting multiple sclerosis has also been reported to cause elevated levels of CXCL13 in the CSF (45).

These observations, coupled with polymerase chain reaction-based findings in patients with early nervous system Lyme disease (10), and the frequent description of increased immunoglobulin production including the presence of oligoclonal bands in CSF of neuroborreliosis patients, suggest that the spirochetes invade the central nervous system very early in infection and elicit a vigorous innate immunity-triggered B cell response, leading to prolonged antibody production.

\section{Treatment, evolution an reinfection}

Three antibiotic regimens have shown their efficacy in neuroborreliosis. In Europe Ceftriaxone iv 2g/day for 21-28 days remains of first intention. The Infectious Diseases Society of America and the American Academy of Neurology recommend a treatment duration of only 14 days, due to the lack of supporting evidence that a one month treatment is superior to a shorter, two weeks, regimen. In Europe, other options include Peniciline G 18-24 MUI/day and Doxycycline $200 \mathrm{mg} /$ day for an equal 21-28 days. Peniciline G is not recommended by the American Guidelines for neurologic Lyme disease. Resolution of symptoms under treatment can be rapid (within the first $48 \mathrm{~h}$ ) or delayed, after the first two weeks of antibiotic therapy. $90 \%$ of acute forms improve under treatment versus $40-50 \%$ of chronic forms (6). Facial paralysis or myelitis can persist as residual neurologic impairment after the end of treatment (9). As noted above, follow-up be serologic testing is of no use, as antibodies can still be detected after cure, even though they do not provide any kind of protection for reinfection.

One single study proved the efficacy of a single dose flash therapy of 200mg of Doxycycline in the $72 \mathrm{~h}$ that follow a tick bite in endemic areas (46). Taking into account the scare data available, the potential for adverse effects and cross resistance, the Lyme consensus conference of 2006 recommends the use of this strategy only in endemic areas and only if the infecting tick has remained attached for more than 48 hours (47).

\section{References}

[1] Blanc F; Gebly. Neurologic and psychiatric manifestations of Lyme disease Med Mal Infect. 2007 Jul-Aug;37(7-8):435-45. Epub 2007 Mar 9

[2] Tranchant C, Warter JM. Lyme borreliosis, Rev Neurol (Paris). 2003 Jan;159(1):23-30

[3] Halperin JJ, Krupp LB, Golightly MG, Volkman DJ, Lyme borreliosis-associated encephalopathy. Neurology. 1990;40(9):1340

[4] Halperin, JJ, Heyes, MP, Keller, TL, Whitman, M. Neuroborreliosis - encephalopathy vs encephalitis. Proceedings of the Vth International Conference on Lyme Borreliosis, Arlington, Virginia, May 1992. Abstr. 1. 1992:1.

[5] Halperin JJ, Nervous system Lyme disease, 2011 UpToDate, Inc, Last update juin 10, 2011 
[6] Hansen K, Lebech AM. The clinical and epidemiological profile of Lyme neuroborreliosis in Denmark 1985-1990. A prospective study of 187 patients with Borrelia burgdorferi specific intrathecal antibody production Brain. 1992 Apr;115 ( Pt 2):399-423

[7] Oschmann P, Dorndorf W, Hornig C, Schäfer C, Wellensiek HJ, Pflughaupt KW. Stages and syndromes of neuroborreliosis. J Neurol. 1998 May;245(5):262-72.

[8] Strle F, Ruzić-Sabljić E, Cimperman J, Lotric-Furlan S, Maraspin V. Comparison of findings for patients with Borrelia garinii andBorrelia afzelii isolated from cerebrospinal fluid, Clin Infect Dis. 2006 Sep 15;43(6):704-10. Epub 2006 Aug 8

[9] De Seze J, Blanc F, Lyme neuroborreliosis: epidemiology, diagnosis and treatment, Lettre Infectiologue, XXVI (3) Mai-Juin 2011, 88-94

[10] Luft BJ, Steinman CR, Neimark HC, et al. Invasion of the central nervous system by Borrelia burgdorferi in acute disseminated infection. JAMA 1992; 267:1364.

[11] Logigian EL, Kaplan RF, Steere AC. Chronic neurologic manifestations of Lyme disease. N Engl J Med 1990; 323:1438.

[12] Halperin JJ, Luft BJ, Anand AK, et al. Lyme neuroborreliosis: central nervous system manifestations. Neurology 1989; 39:753.

[13] Logigian EL, Steere AC. Clinical and electrophysiologic findings in chronic neuropathy of Lyme disease. Neurology 1992; 42:303.

[14] Rupprecht TA, Koedel U, Fingerle V, Pfister HW. The pathogenesis of lyme neuroborreliosis: from infection to inflammation. Mol Med 2008; 14:205.

[15] Stanek G, Strle F, Lyme disease: European perspective. Infect Dis Clin North Am. 2008;22(2):327

[16] Halperin JJ, Golightly M. Lyme borreliosis in Bell's palsy. Long Island Neuroborreliosis Collaborative Study Group. Neurology 1992; 42:1268.

[17] Blanc F, Jaulhac B, Fleury M, de Seze J, de Martino SJ, Remy V, Blaison G, Hansmann Y,Christmann D, Tranchant C Relevance of the antibody index to diagnose Lymeneuroborreliosis among seropositive patients, Neurology. 2007 Sep 4;69(10):953-8.

[18] Rothermel H, Hedges TR 3rd, Steere AC Optic neuropathy in children with Lyme disease. Pediatrics. 2001;108(2):477

[19] Belman AL, Iyer M, Coyle PK, Dattwyler R Neurologic manifestations in children with North American Lyme disease, Neurology. 1993;43(12):2609

[20] Blanc F, Froelich S, Vuillemet F, Carré S, Baldauf E, de Martino S, Jaulhac B, Maitrot D,Tranchant C, de Seze J., Acute myelitis and Lyme disease, Rev Neurol (Paris). 2007 Nov;163(11):1039-47

[21] Sibony P, Halperin J, Coyle PK, Patel K. Reactive Lyme serology in optic neuritis, J Neuroophthalmol. 2005 Jun;25(2):71-82

[22] Krim E, Guehl D, Burbaud P, Lagueny A., Retrobulbar optic neuritis: a complication of Lyme disease? J Neurol Neurosurg Psychiatry. 2007 Dec;78(12):1409-10

[23] Blanc F, Ballonzoli L, Marcel C, De Martino S, Jaulhac B, de Seze J. Lyme optic neuritis, J Neurol Sci. 2010 Aug 15;295(1-2):117-9. Epub 2010 Jun

[24] Sparsa L, Blanc F, Lauer V, Cretin B, Marescaux C, Wolff V Recurrent ischemic strokes revealing Lymemeningovascularitis Rev Neurol (Paris). 2009 Mar;165(3):273-7. Epub 2008 Aug 28 
[25] Oksi J, Kalimo H, Marttila RJ, Marjamäki M, Sonninen P, Nikoskelainen J, Viljanen MK,Inflammatory brain changes in Lyme borreliosis. A report on three patients and review of literature. Brain. 1996 Dec;119 ( Pt 6):2143-54

[26] Krupp LB, Masur D, Schwartz J, Coyle PK, Langenbach LJ, Fernquist SK, Jandorf L, Halperin JJ, Cognitive functioning in late Lyme borreliosis Arch Neurol. 1991;48(11):1125

[27] Logigian EL, Kaplan RF, Steere AC. Chronic neurologic manifestations of Lyme disease. N Engl J Med 1990; 323:1438

[28] Klempner MS, Hu LT, Evans J, et al. Two controlled trials of antibiotic treatment in patients with persistent symptoms and a history of Lyme disease. N Engl J Med 2001; 345:85.

[29] Fallon BA, Keilp JG, Corbera KM, et al. A randomized, placebo-controlled trial of repeated IV antibiotic therapy for Lyme encephalopathy. Neurology 2008; 70:992.

[30] Halperin JJ. Prolonged Lyme disease treatment: enough is enough. Neurology 2008; 70:986.

[31] Feder HM Jr, Johnson BJ, O'Connell S, et al. A critical appraisal of "chronic Lyme disease". N Engl J Med 2007; 357:1422.

[32] Blanc F. Epidemiology of Lyme borreliosis and neuroborreliosis in France Rev Neurol (Paris). 2009 Aug-Sep;165(8-9):694-701. Epub 2009 May 17

[33] Stiernstedt GT, Granström M, Hederstedt B, Sköldenberg B. Diagnosis of spirochetal meningitis by enzyme-linked immunosorbent assay and indirect immunofluorescence assay in serum and cerebrospinal fluid. J Clin Microbiol 1985; 21:819.

[34] Steere AC, Berardi VP, Weeks KE, et al. Evaluation of the intrathecal antibody response to Borrelia burgdorferi as a diagnostic test for Lyme neuroborreliosis. J Infect Dis 1990; 161:1203.

[35] Ljøstad U, Skarpaas T, Mygland A. Clinical usefulness of intrathecal antibody testing in acute Lyme neuroborreliosis. Eur J Neurol 2007; 14:873.

[36] Halperin JJ, Volkman DJ, Wu P. Central nervous system abnormalities in Lyme neuroborreliosis. Neurology 1991; 41:1571.

[37] Pícha D, Moravcová L, Zdárský E, Benes J. Clinical comparison of immunoblot and antibody index for detection of intrathecal synthesis of specific antibodies in Lymeneuroborreliosis Eur J Clin Microbiol Infect Dis. 2000 Oct;19(10):805-6.

[38] Baig S, Olsson T, Hansen K, Link H. Anti-Borrelia burgdorferi antibody response over the course of Lyme neuroborreliosis. Infect Immun 1991; 59:1050.

[39] Lakos A. CSF findings in Lyme meningitis. J Infect 1992; 25:155.

[40] Hansen K, Cruz M, Link H. Oligoclonal Borrelia burgdorferi-specific IgG antibodies in cerebrospinal fluid in Lyme neuroborreliosis. J Infect Dis 1990; 161:1194.

[41] Nocton JJ, Bloom BJ, Rutledge BJ, et al. Detection of Borrelia burgdorferi DNA by polymerase chain reaction in cerebrospinal fluid in Lyme neuroborreliosis. J Infect Dis 1996; 174:623.

[42] Tumani H, Cadavid D. Are high CSF levels of CXCL13 helpful for diagnosis of Lyme neuroborreliosis? Neurology 2011; 76:1034.

[43] Marra CM, Tantalo LC, Sahi SK, et al. CXCL13 as a cerebrospinal fluid marker for neurosyphilis in HIV-infected patients with syphilis. Sex Transm Dis 2010; 37:283. 
[44] Schmidt C, Plate A, Angele B, et al. A prospective study on the role of CXCL13 in Lyme neuroborreliosis. Neurology 2011; 76:1051.

[45] Rupprecht TA, Pfister HW, Angele B, et al. The chemokine CXCL13 (BLC): a putative diagnostic marker for neuroborreliosis. Neurology 2005; 65:448.

[46] Nadelman RB, Nowakowski J, Fish D, Falco RC, Freeman K, McKenna D, Welch P, Marcus R, Agüero-Rosenfeld ME, Dennis DT, Wormser GP; Tick Bite Study Group Prophylaxis with single-dose doxycycline for the prevention of Lymedisease after an Ixodes scapularis tick bite. N Engl J Med. 2001 Jul 12;345(2):79-84

[47] Clavelou P.Consensus conference on Lyme borreliosis.Presse Med. 2008 Dec;37(12):1707-8. Epub 2008 Nov 1 


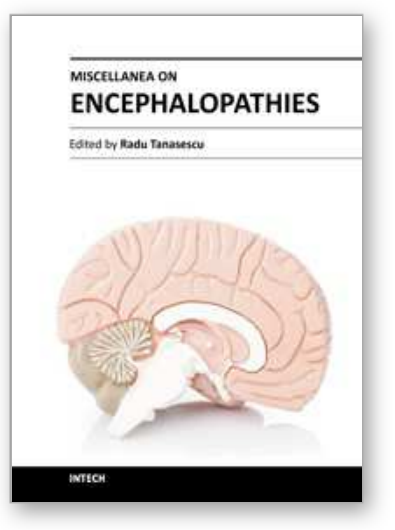

\author{
Miscellanea on Encephalopathies \\ Edited by Dr. Radu Tanasescu
}

ISBN 978-953-51-0499-5

Hard cover, 202 pages

Publisher InTech

Published online 18, April, 2012

Published in print edition April, 2012

The book project "Miscellanea on Encephalopathies" aims to cover some of the important aspects of infectious-related encephalopathies, post-transplantation and drug-induced encephalopathies, by transmitting valuable information filtered through the real life clinical and research experience of the authors.

\title{
How to reference
}

In order to correctly reference this scholarly work, feel free to copy and paste the following:

Ruxandra Calin, Adriana Hristea and Radu Tanasescu (2012). Central Nervous System Involvement in Lyme Disease - Making the Diagnosis and Choosing the Correct Treatment, Miscellanea on Encephalopathies, Dr. Radu Tanasescu (Ed.), ISBN: 978-953-51-0499-5, InTech, Available from:

http://www.intechopen.com/books/miscellanea-on-encephalopathies/central-neurological-manifestations-oflyme-disease-encephalopathy-and-more

\section{INTECH}

open science | open minds

\author{
InTech Europe \\ University Campus STeP Ri \\ Slavka Krautzeka 83/A \\ 51000 Rijeka, Croatia \\ Phone: +385 (51) 770447 \\ Fax: +385 (51) 686166 \\ www.intechopen.com
}

\author{
InTech China \\ Unit 405, Office Block, Hotel Equatorial Shanghai \\ No.65, Yan An Road (West), Shanghai, 200040, China \\ 中国上海市延安西路65号上海国际贵都大饭店办公楼 405 单元 \\ Phone: +86-21-62489820 \\ Fax: $+86-21-62489821$
}


(C) 2012 The Author(s). Licensee IntechOpen. This is an open access article distributed under the terms of the Creative Commons Attribution 3.0 License, which permits unrestricted use, distribution, and reproduction in any medium, provided the original work is properly cited. 